\title{
Emergent Rhombus Tilings from Molecular Interactions with $M$-fold Rotational Symmetry
}

\author{
Stephen Whitelam, ${ }^{1,}$ Isaac Tamblyn, ${ }^{2}$ Juan P. Garrahan, ${ }^{3}$ and Peter H. Beton ${ }^{3}$ \\ ${ }^{1}$ Molecular Foundry, Lawrence Berkeley National Laboratory, 1 Cyclotron Road, Berkeley, California 94720, USA \\ ${ }^{2}$ Department of Physics, University of Ontario Institute of Technology, Oshawa, Ontario L1H 7K4, Canada \\ ${ }^{3}$ School of Physics and Astronomy, University of Nottingham, Nottingham NG7 2RD, United Kingdom
}

(Received 15 November 2014; published 20 March 2015)

\begin{abstract}
We show that model molecules with particular rotational symmetries can self-assemble into network structures equivalent to rhombus tilings. This assembly happens in an emergent way, in the sense that molecules spontaneously select irregular fourfold local coordination from a larger set of possible local binding geometries. The existence of such networks can be rationalized by simple geometrical arguments, but the same arguments do not guarantee a network's spontaneous self-assembly. This class of structures must in certain regimes of parameter space be able to reconfigure into networks equivalent to triangular tilings.
\end{abstract}

DOI: 10.1103/PhysRevLett.114.115702

PACS numbers: 64.75.Yz, 05.70.Fh, 81.16.Fg

The self-assembly of molecules at surfaces has practical application because it allows us to influence the properties of those surfaces [1,2]. It is also of fundamental interest: self-assembly at surfaces has provided insight into the general question of how to encourage the self-assembly of a desired structure while avoiding the self-assembly of undesired structures [3-7]. In several cases, basic considerations of molecular symmetry and geometry have been shown to be key to understanding the outcome of selfassembly at surfaces. For example, a wide range of molecules whose atomic details differ but whose interactions possess threefold rotational symmetry form honeycomb networks and polygon variants thereof, both in and out of equilibrium [8-13]. Molecules whose binding geometries are equivalent to those of rhombus tiles form rhombus tilings of the plane [14-17].

In those examples, the restriction of preferred molecular binding geometries to only those characteristic of a particular network encourages formation of that network and helps prevent the formation of other possible networks. Here we demonstrate in simulations an example of network self-assembly that happens instead in an emergent fashion. Collections of mutually attractive model molecules having particular rotational symmetries spontaneously select only a subset of possible local binding geometries. By doing so, they self-assemble into extended networks equivalent to rhombus tilings of the plane [18-24].

To provide context for this result we consider in Fig. 1 the regular Platonic tilings, which are coverings of the plane by nonoverlapping regular triangular, square, and hexagonal tiles [25]. The vertices of such tilings possess sixfold, fourfold, and threefold rotational symmetry, respectively. Other authors have shown that the networks equivalent to such tilings can be realized by the selfassembly of model molecules whose interactions possess the appropriate rotational symmetries [26-28]. By way of illustration, we show pictures in the figure of such networks and the equivalent tilings, self-assembled in our simulations from collections of "patchy particles" $[29,30]$ in two dimensions. The particles in question are hard disks that

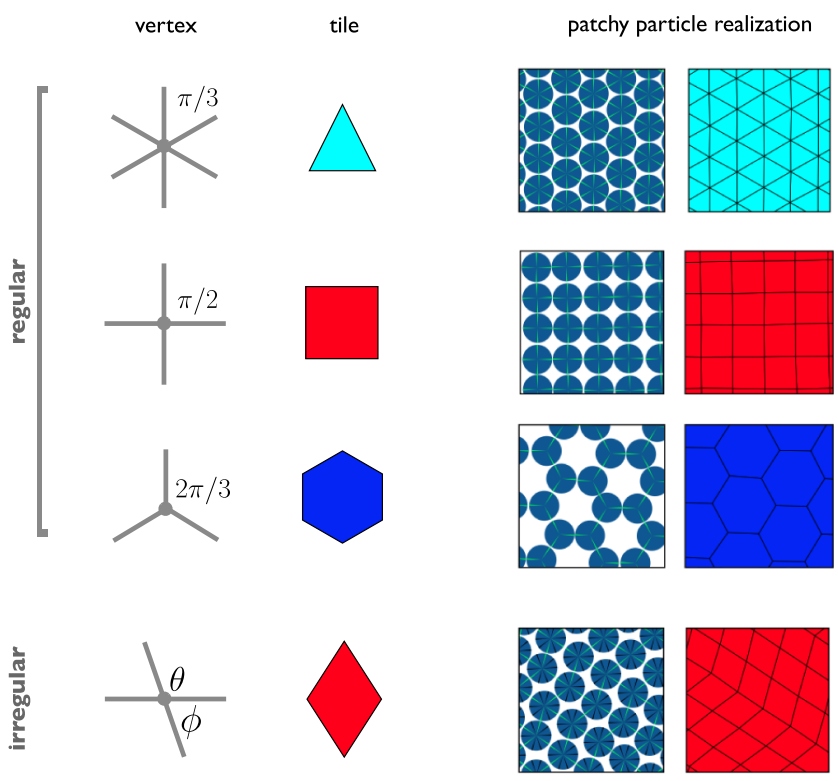

FIG. 1 (color online). Platonic tilings of triangles, squares, or hexagons result from regular six-vertices, four-vertices, or threevertices, respectively, and can self-assemble from collections of building blocks with the corresponding rotational symmetries [26-28]. The four-vertex can be made irregular and results in a tile, a rhombus, whose sides are of equal length. As we show in this Letter, irregular four-vertex networks equivalent to rhombus tilings spontaneously self-assemble from building blocks with $M$-fold rotational symmetry, where $M \geq 4$ is even and not a multiple of six. 
possess $M$-fold rotational symmetry of their interactions: disks attract each other in a pairwise fashion via $M$ regularly placed "patches" of opening angle $2 w$. Two engaged patches result in a favorable binding energy $-\epsilon k_{B} T$. Disk interactions are "square well" in both angle [31] and range; see Sec. S1. By slowly cooling collections of such disks (Sec. S2) we observe the spontaneous assembly of the networks shown.

Thermodynamically, it is clear why disks form these networks and not other ones. Disks within these networks have all their patches engaged and so possess the least possible energy. Networks are also geometrically unstrained -on average, disk patch bisectors point along the lines connecting vertices - and so disks retain as much rotational and vibrational entropy as possible. Lack of strain is possible because the rotational symmetries of the network vertices and disk interactions are identical, and because the resulting tile has all sides of equal length, enabling its formation by disks possessing a single preferred (average) interaction distance. Dynamically, assembly is successful because slow cooling allows disks to select low-energy local binding environments before interactions become strong enough to cause kinetic trapping.

Given that the networks equivalent to the Platonic tilings are built from regular vertices and tiles, it is natural to ask if one can arrange for the self-assembly of irregular variants of these networks. The six-vertex, if deformed, gives rise (locally) to triangles whose sides are not all of equal length. A single type of molecule could therefore not form a network of such vertices without strain, unless it possessed multiple preferred interaction ranges (see, e.g., Refs. [32,33]). But the four-vertex is different. As shown in Fig. 1, a four-vertex harboring two types of internal angle $\phi$ and $\theta$ is equivalent to a rhombus tile possessing these two internal angles. A rhombus has all sides equal, and so, in principle, a single model molecule with the appropriate rotational symmetry and a single preferred interaction range could form an unstrained network equivalent to a tiling of such rhombuses.

This is what we show in the lower right-hand panels of Fig. 1. However, the model molecule in question possesses not irregular fourfold rotational symmetry but regular tenfold rotational symmetry. Cooling collections of this molecule (with patches of half-width $w=5^{\circ}$ ) results in self-assembly of the pattern shown. In this pattern, disks engage only four of their ten patches: the irregular fourvertex has emerged spontaneously. By comparing the left and center panels of Fig. 2(a), which show a larger piece of this network, we see that domains of parallel-pointing rhombuses result when disks engage four patches whose (a)
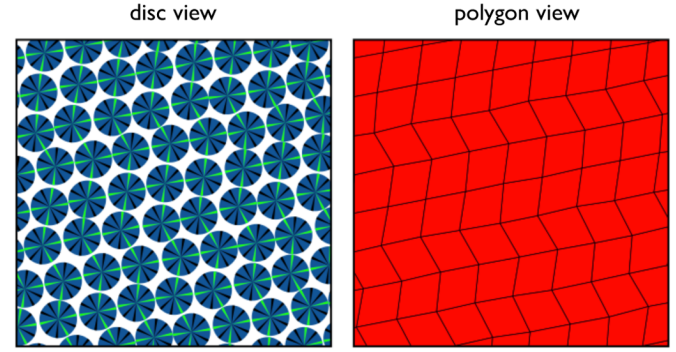

(c)
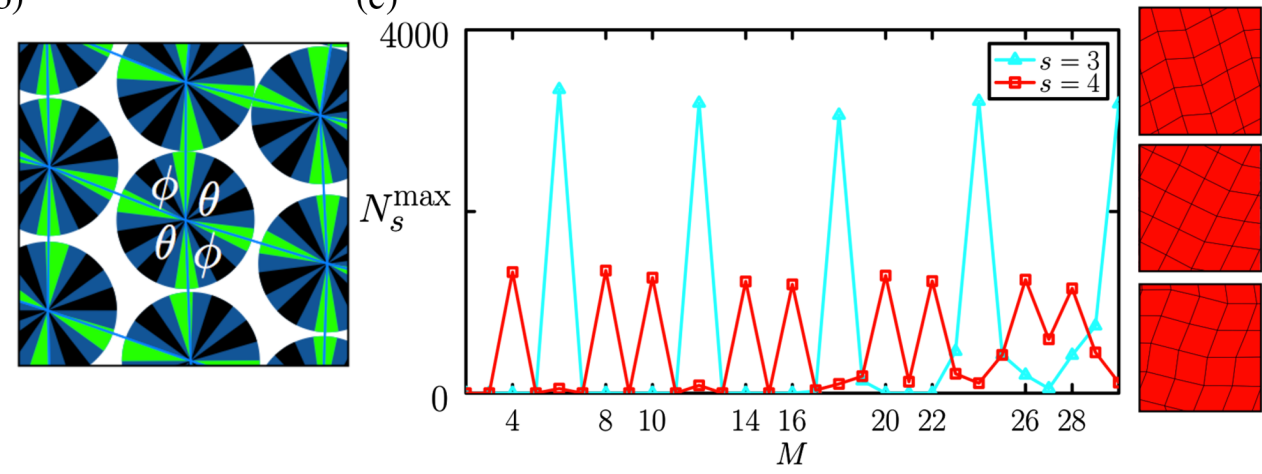

14

20

26

FIG. 2 (color online). Rhombus tilings emerge from particular regular rotational symmetries of building block interactions. (a) Region of rhombus tiling self-assembled from ten-patch disks $\left(w=5^{\circ}\right)$, in disk (left) and polygon (center) views. This tiling is similar to the "tilted" rhombus phase [14,16,34] (right) that results from the self-assembly of mutually attractive rhombus tiles with internal angles $2 \pi / 5$ and $3 \pi / 5$. (b) Geometry used to motivate the observation that unstrained rhombus tilings can form from regular $M$-patch disks, where $M$ is even and not a multiple of six. (c) In simulations, disks of this nature spontaneously self-assemble into rhombus tilings if cooled slowly. Here we plot the largest number of convex polygons of size $s=3$ or 4 obtained upon cooling collections of $M$-patch disks with $w=2^{\circ}$. Inspection of configurations reveals the four-gons at the labeled values of $M$ to be rhombuses (see snapshots right and Figs. S2 and S3.) 
bisectors are separated by angles $(2,3,2,3) \times \pi / 5$, in order. Boundaries between parallel-pointing domains result when engaged patch bisectors possess angular separations ordered instead as $(2,2,3,3) \times \pi / 5$. The resulting tiling is similar to that made by the self-assembly of rhombusshaped tiles possessing internal angles $2 \pi / 5$ and $3 \pi / 5$ (right; see Sec. S3).

Thus, collections of model molecules with tenfold rotational symmetry spontaneously select local irregular fourfold coordination and so self-assemble into an extended network. This example turns out to be a particular case of a more general phenomenon, as we now describe. We can ask from what regular rotational symmetries are rhombus tilings possible geometrically, focusing on the narrow-patch limit in which patch bisectors must point along the lines connecting network vertices. In Fig. 2(b) we show a picture of a ten-patch disk that forms the vertex of a self-assembled rhombus tiling (for the purposes of the following argument we shall ignore that fact that disk patches in this picture are of finite width). The angles $\phi$ and $\theta$ are the two internal angles of the rhombus. Because these angles meet at a vertex, they satisfy

$$
2(\phi+\theta)=2 \pi
$$

Imagine now that this disk has not ten but $M$ patches, and let the angles $\phi$ and $\theta$ correspond to the intervals separating an integer numbers of patches, say, $\alpha$ and $\beta$, respectively. That is, let $\phi=2 \pi \alpha / M$ and $\theta=2 \pi \beta / M$. Inserting these expressions into Eq. (1) gives

$$
\alpha+\beta=M / 2 .
$$

Because $\alpha$ and $\beta$ are integers, $M / 2$ must be an integer, and so $M$ must be even. Thus, it is geometrically possible for disks with even-numbered rotational symmetry to form unstrained rhombus tilings. Thinking now of the potential self-assembly of such tilings, we note that if $M$ is a multiple of six then disks can also form an unstrained triangular tiling, and this-being made from six-vertices rather than four-vertices-will be energetically preferred to the rhombus tiling. Simple geometric arguments therefore suggest the possibility of having rhombus tilings self-assemble from model molecules with $M$-fold rotational symmetry, where $M(\geq 4)$ is even and not a multiple of six.

In Fig. 2(c) we show using simulations of $M$-patch disks that this possibility can be realized: collections of such molecules do self-assemble into rhombus tilings if cooled slowly. We plot the largest number $N_{s}^{\max }$ of polygons (drawn atop networks; see Sec. S1) having $s$ sides that were seen over the course of long cooling simulations. Networks self-assembled at the labeled values of $M$ contain numerous four-gons. Inspection of networks (see, e.g., snapshots at right of Fig. 2(c) and Fig. S2) reveals these four-gons to be rhombuses, arranged into rhombus tilings. The tilings predicted by geometry to exist are therefore kinetically accessible: disks self-assemble into rhombus tilings in preference to any of the many possible disordered networks.

Rhombuses in tilings can also be of more than one shape, consistent with the fact that Eq. (2) constrains only the sum of $\alpha$ and $\beta$ : an $M$-patch disk can in general make rhombuses of more than one type $(\alpha, M / 2-\alpha)$, by which we mean a rhombus with internal angles $(\alpha, M / 2-\alpha) \times 2 \pi / M$. Taking $\alpha \leq \beta$, sterically allowed, strain-free rhombuses are characterized by integers $\alpha$ satisfying $M / 6<\alpha \leq M / 4$ [steric constraints alone, for the disk parameters used in this work (see Sec. S1), dictate a lesser lower bound of $\alpha>(M / \pi) \arcsin (5 / 11) \approx M / 6.66]$. For instance, the ten-patch disk makes only the $(2,3)$ rhombus: the $(1,4)$ rhombus is forbidden sterically. But for 16 patches and upwards, rhombuses in tilings can be of more than one type. For example, the 20-patch disk forms the $(4,6)$ and $(5,5)$ rhombuses [a boundary between these tile types is shown in the middle snapshot of Fig. 2(c)]; the 28-patch disk forms the $(5,9),(6,8)$, and $(7,7)$ rhombuses. We see the sterically allowed, unstrained rhombuses in roughly equal numbers in our simulations, indicating that they are freeenergetically equivalent. Tilings that result from cooling simulations are also imperfect, in the sense that they contain nonrhombic grain boundaries that result from the dynamic process of self-assembly (the more so as $M$ increases).

These emergent rhombus tilings must be polymorphic with at least one other solid phase in certain regimes of parameter space. To see this, we note than in simulations thus far we have focused on model molecules with specific orientational interactions, i.e., narrow patches, because rhombus tilings are free of geometrical strain and so can, in principle, form from disks whose patches are of infinitesimal width (we checked that the expected rhombus tilings self-assemble, for $M \leq 32$, for the finite but narrow half-width $\left.w=0.5^{\circ}\right)$. However, real molecules possess finite flexibility of binding and so are better modeled by disks whose patches have appreciable width $[13,35]$. In the limit of wide patches, when the whole circumference of the disk is sticky (i.e., when $w=\pi / M$ ), networks equivalent to triangular tilings must result. Somewhere between the wide- and narrow-patch limits, then, polymorphism must occur.

We verified that this is so for ten-patch disks. In Fig. 3(a) we show that cooling collections of ten-patch disks with patches of half-width $w \lesssim 10^{\circ}$ results in tilings of four-gons (which we verified by eye to be rhombuses), while disks with wider patches form triangular tilings. $N_{s}^{\max }$ again denotes the largest number of $s$-gons observed over the course of long cooling simulations. Triangular tilings for $w \approx 10^{\circ}$ are irregular and become regular as $w$ approaches $\pi / 10=18^{\circ}$. Near $w=10^{\circ}$ we observe polymorphism: the first tiling to assemble is the rhombus one, which, as temperature is reduced (as $\epsilon$ is increased), converts to a triangular tiling. 
(a)

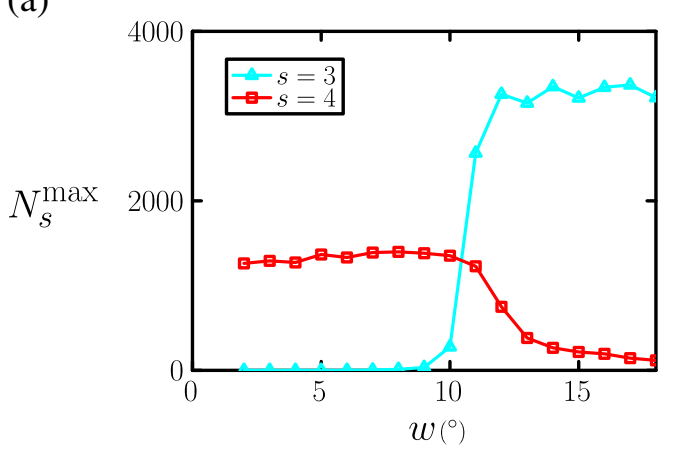

(b)

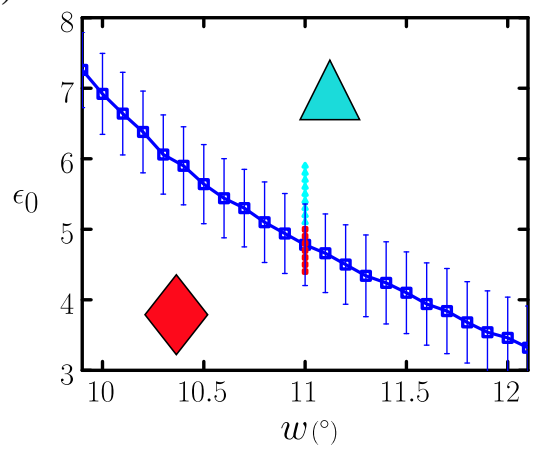

(c)

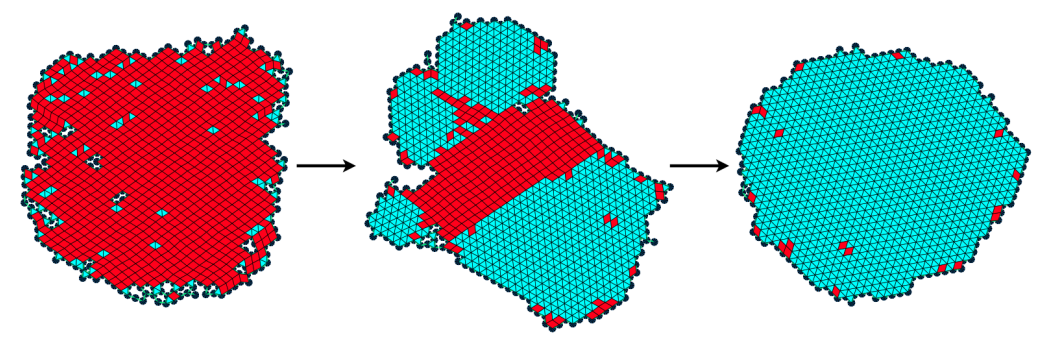

FIG. 3 (color online). Rhombus-triangle polymorphism. (a) Cooling collections of ten-patch disks result in self-assembly of the rhombus phase when interactions are orientationally specific and of the triangle phase as interactions approach the isotropic limit. Between these limits the two phases can coexist and interconvert, with rhombuses favored by entropy and triangles by energy (see also Fig. S4). (b) The rhombus-triangle phase boundary calculated at zero pressure using mean-field theory [Eq. (S6)]. Small symbols for $w=11^{\circ}$ describe the results of direct coexistence simulations, which are consistent with the mean-field estimate (see Sec. S4). (c) Rhombus-to-triangle transformation at parameter set $\left(\epsilon=7, w=11^{\circ}\right)$, where Eq. (S6) predicts the bulk free-energy density of the triangle phase to be $2.2 k_{B} T$ less than that of the rhombus phase.

This conversion occurs because disks in networks equivalent to rhombus tilings are unstrained geometrically and so possess more rotational and translational entropy than do disks in strained triangular tilings. But disks forming triangular tilings make six contacts rather than four and so are energetically preferred. Triangles therefore emerge at low temperature. A related energy-versusentropy competition between open and close-packed lattices has been demonstrated for patchy silica spheres [36]. In Fig. 3 we show the phase boundary between triangle and rhombus tilings, which we calculated at zero pressure using mean-field theory (see Sec. S4). Above the line $\epsilon_{0}(w)$, triangular tilings are lower in free energy than rhombus tilings [37]. Figure 3(c) shows a rhombus-to-triangle tiling transformation involving disks of patch half-width $w=11^{\circ}$. We therefore predict that emergent rhombus tilings formed by molecules with sufficient flexibility of binding should reconfigure in response to changes of temperature.

We note finally that the Archimedean tilings, which are made from regular vertices and harbor two tile types, cannot be prepared in an unstrained manner using disks with regular $M$-fold symmetry. Self-assembly of Archimedean tilings can be achieved instead using irregular patch placement [27] or an odd number of suitably wide patches $[28,38,39]$ (or the equivalent effective coordination $[40,41])$; see Sec. S5.
We have shown that model molecules with particular rotational symmetries can self-assemble into rhombus tilings in an emergent way, by spontaneously selecting irregular fourfold local coordination from a larger set of possible local binding motifs. We predict that such tilings, if made from molecules with sufficient binding flexibility, could reconfigure in response to changes of temperature, which may have energy-transfer or phase-change application. Experimentally, cyclic porphyrin polymers [42] can be synthesized with the required rotational symmetries and therefore offer a possible route to the realization of the rhombus tilings identified here [43].

This work was done as part of a User Project at the Molecular Foundry, Lawrence Berkeley National Laboratory, supported by the Director, Office of Science, Office of Basic Energy Sciences, of the U.S. Department of Energy under Contract No. DE-AC02-05CH11231. J.P. G. and P. H. B. acknowledge support from EPSRC Grant No. EP/K01773X/1. I. T. acknowledges support from NSERC.

*swhitelam@lbl.gov

[1] J. A. A. W. Elemans, S. Lei, and S. De Feyter, Angew. Chem., Int. Ed. Engl. 48, 7298 (2009).

[2] L. Bartels, Nat. Chem. 2, 87 (2010). 
[3] G. M. Whitesides and B. Grzybowski, Science 295, 2418 (2002).

[4] D. Philp and J. F. Stoddart, Angew. Chem., Int. Ed. Engl. 35, 1154 (1996).

[5] M. F. Hagan and D. Chandler, Biophys. J. 91, 42 (2006).

[6] A. W. Wilber, J. P. Doye, A. A. Louis, E. G. Noya, M. A. Miller, and P. Wong, J. Chem. Phys. 127, 085106 (2007).

[7] D. C. Rapaport, Phys. Biol. 7, 045001 (2010).

[8] A. K. Geim and K. S. Novoselov, Nat. Mater. 6, 183 (2007).

[9] Y. He, Y. Chen, H. Liu, A. E. Ribbe, and C. Mao, J. Am. Chem. Soc. 127, 12202 (2005).

[10] L. Lichtenstein, M. Heyde, and H.-J. Freund, Phys. Rev. Lett. 109, 106101 (2012).

[11] M. Bieri, M. Treier, J. Cai, K. Aït-Mansour, P. Ruffieux, O. Gröning, P. Gröning, M. Kastler, R. Rieger, X. Feng et al., Chem. Commun. (Cambridge) 45, 6919 (2009).

[12] C.-A. Palma, P. Samorì, and M. Cecchini, J. Am. Chem. Soc. 132, 17880 (2010).

[13] S. Whitelam, I. Tamblyn, T. K. Haxton, M. B. Wieland, N. R. Champness, J. P. Garrahan, and P. H. Beton, Phys. Rev. X 4, 011044 (2014).

[14] M. O. Blunt, J. C. Russell, M. C. Giménez-López, J. P. Garrahan, X. Lin, M. Schröder, N. R. Champness, and P. H. Beton, Science 322, 1077 (2008).

[15] M. O. Blunt, X. Lin, M. del Carmen Gimenez-Lopez, M. Schröder, N. R. Champness, and P. H. Beton, Chem. Commun. (Cambridge) 20, 2304 (2008).

[16] A. Stannard, M. O. Blunt, P. H. Beton, and J. P. Garrahan, Phys. Rev. E 82, 041109 (2010).

[17] S. Whitelam, I. Tamblyn, P. H. Beton, and J. P. Garrahan, Phys. Rev. Lett. 108, 035702 (2012).

[18] M. E. Fisher, Phys. Rev. 124, 1664 (1961).

[19] P. W. Kasteleyn, J. Math. Phys. (N.Y.) 4, 287 (1963).

[20] H. Blöte and H. Hilhorst, J. Phys. A 15, L631 (1982).

[21] C. L. Henley, Quasicrystals: The State of the Art edited by D. P. DiVincenzo, and P. J. Steinhardt, Vol. 429 (World Scientific, Singapore, 1991).

[22] H. Cohn, R. Kenyon, and J. Propp, J. Am. Math. Soc. 14, 297 (2001).

[23] N. Destainville, M. Widom, R. Mosseri, and F. Bailly, J. Stat. Phys. 120, 799 (2005).

[24] F. Alet, Y. Ikhlef, J. L. Jacobsen, G. Misguich, and V. Pasquier, Phys. Rev. E 74, 041124 (2006).

[25] B. Grfinbaum and G. Shephard, Tilings and Patterns (Freeman, New York, 1987).

[26] G. Doppelbauer, E. Bianchi, and G. Kahl, J. Phys. Condens. Matter 22, 104105 (2010).
[27] M. Antlanger, G. Doppelbauer, and G. Kahl, J. Phys. Condens. Matter 23, 404206 (2011).

[28] J. P. Doye, A. A. Louis, I.-C. Lin, L. R. Allen, E. G. Noya, A. W. Wilber, H. C. Kok, and R. Lyus, Phys. Chem. Chem. Phys. 9, 2197 (2007).

[29] Z. Zhang and S. C. Glotzer, Nano Lett. 4, 1407 (2004).

[30] P. Tartaglia and F. Sciortino, J. Phys. Condens. Matter 22, 104108 (2010).

[31] N. Kern and D. Frenkel, J. Chem. Phys. 118, 9882 (2003).

[32] M. C. Rechtsman, F. H. Stillinger, and S. Torquato, Phys. Rev. E 73, 011406 (2006).

[33] M. C. Rechtsman, F. H. Stillinger, and S. Torquato, Phys. Rev. Lett. 95, 228301 (2005).

[34] X. Ye, J. Chen, M. Engel, J. A. Millan, W. Li, L. Qi, G. Xing, J. E. Collins, C. R. Kagan, J. Li et al., Nat. Chem. 5, 466 (2013).

[35] I. Saika-Voivod, F. Smallenburg, and F. Sciortino, J. Chem. Phys. 139, 234901 (2013).

[36] X. Mao, Q. Chen, and S. Granick, Nat. Mater. 12, 217 (2013).

[37] The value of $w$ at which polymorphism occurs decreases with increasing $M$ : it is about $10^{\circ}$ for 10 patches and $1^{\circ}$ for 32 patches.

[38] M. N. van der Linden, J. P. Doye, and A. A. Louis, J. Chem. Phys. 136, 054904 (2012).

[39] A. Reinhardt, F. Romano, and J. P. K. Doye, Phys. Rev. Lett. 110, 255503 (2013).

[40] D. Écija, J. I. Urgel, A. C. Papageorgiou, S. Joshi, W. Auwärter, A. P. Seitsonen, S. Klyatskaya, M. Ruben, S. Fischer, S. Vijayaraghavan et al., Proc. Natl. Acad. Sci. U.S.A. 110, 6678 (2013).

[41] J. A. Millan, D. Ortiz, G. van Anders, and S. C. Glotzer, ACS Nano 8, 2918 (2014).

[42] D. V. Kondratuk, L. M. A. Perdigao, A. M. S. Esmail, J. N. O'Shea, P. H. Beton, and H. L. Anderson, Nat. Chem. (in press).

[43] See Supplemental Material at http://link.aps.org/ supplemental/10.1103/PhysRevLett.114.115702, which includes Refs. [44-47], for additional simulation details.

[44] S. Whitelam, E. H. Feng, M. F. Hagan, and P. L. Geissler, Soft Matter 5, 1251 (2009).

[45] B. Chen and J. I. Siepmann, J. Phys. Chem. B 105, 11275 (2001).

[46] J. Geng and J. V. Selinger, Phys. Rev. E 80, 011707 (2009).

[47] S. Whitelam, Phys. Rev. Lett. 105, 088102 (2010). 